\title{
HYPERSPACES OF TOPOLOGICAL VECTOR SPACES: THEIR EMBEDDING IN TOPOLOGICAL VECTOR SPACES
}

\author{
PREM PRAKASH AND MURAT R. SERTEL
}

\begin{abstract}
Let $L$ be a real (Hausdorff) topological vector space. The space $\mathscr{K}[L]$ of nonempty compact subsets of $L$ forms a (Hausdorff) topological semivector space with singleton origin when $\mathscr{K}[L]$ is given the uniform (equivalently, the finite) hyperspace topology determined by $L$. Then $\mathcal{K}[L]$ is locally compact iff $L$ is so. Furthermore, $K_{2}[L]$, the set of nonempty compact convex subsets of $L$, is the largest pointwise convex subset of $\mathscr{K}[L]$ and is a cancellative topological semivector space. For any nonempty compact and convex set $X \subset L$, the collection $K_{2}[X] \subset$ K2 $[L]$ is nonempty compact and convex. $L$ is iseomorphically embeddable in $K_{2}[L]$ and, in turn, there is a smallest vector space $\mathcal{L}$ in which $K_{2}[L]$ is algebraically embeddable (as a cone). Furthermore, when $L$ is locally convex, $\mathcal{L}$ can be given a locally convex vector topology $\mathscr{T}$ such hat the algebraic embedding of $K_{2}[L]$ in $\mathcal{E}$ is an iseomorphism, and then $\mathcal{L}$ is normable iff $L$ is so; indeed, $\mathcal{T}$ can be so chosen that, when $L$ is normed, the embedding of $L$ in $K_{2}[L]$ and that of $\operatorname{K2}[L]$ in $\mathfrak{L}$ are both iseometries.
\end{abstract}

1. Preliminaries. $R$ denotes the set of real numbers with the usual topology, and $R_{+}=\{\lambda \in R \mid \lambda \geqslant 0\}$. For any set $X,[X]$ denotes the set of nonempty subsets of $X$. When $X$ is a topological space, $\mathscr{K}[X]$ denotes the set of compact nonempty subsets of $X$. When $X$ lies in a real vector space, $2[X]$ denotes the set of convex nonempty subsets of $X$. Finally, when $X$ lies in a real topological vector space, $\mathfrak{K} 2[X]=\mathscr{K}[X] \cap \mathcal{2}[X]$.

In topologizing hyperspaces (i.e., spaces of subsets), we will use the uniform topology, regarding which we adopt Michael [1] as standard reference. Let $X$ be a uniform space, and let $\left\{E_{\alpha} \subset X \times X \mid \alpha \in \mathbb{Q}\right\}$ be a fundamental system of symmetric entourages of $X$. The uniform topology for $[X]$ is the topology generated by declaring $\mathscr{E}_{\alpha}[A]=\left\{B \in[X] \mid B \subset E_{\alpha}(A)\right.$ and $\left.A \subset E_{\alpha}(B)\right\}$ for each $\alpha \in \mathbb{Q}$ to be a nbd of $A \quad(A \in[X])$. By the uniform topology on a hyperspace $\mathcal{H}[X] \subset[X]$ is meant the relative topology of $\mathcal{H}[X]$ when $[X]$ carries the uniform topology.

1.0 Definition [2]. Let $(S, \oplus)$ be a commutative semigroup and $\Psi: R_{+} \times S$ $\rightarrow S$ a map such that, denoting $\Psi(\lambda, s)=\lambda s$,

Received by the editors December 20, 1974 and, in revised form, August 6, 1975.

AMS (MOS) subject classifications (1970). Primary 54B20, 54C25; Secondary 57A17.

Key words and phrases. Topological vector space, topological semivector space, compact convex subsets, hyperspace, locally convex vector space, normable vector space, embedding, iseomorphism, cancellative topological semivector space. 


$$
\begin{aligned}
\lambda(\mu s) & =(\lambda \cdot \mu) s & & \text { (left action), } \\
1 s & =s & & \text { (unitariness), } \\
\lambda(s \oplus t) & =\lambda s \oplus \lambda t & & \text { (homomorphism) }
\end{aligned}
$$

for all $\lambda, \mu \in R_{+}$and $s, t \in S$. We call $S$ a semivector space. When $S$ is a Hausdorff space and the operations $\oplus$ and $\Psi$ are both continuous, we call $S$ a topological semivector space.

Thus, real vector spaces are all semivector spaces, so that the topological vector spaces we speak of are those with Hausdorff topology.

2. Semivector hyperspaces of topological vector spaces. Let $L$ be a real vector space, and $e$ its identity element. Now $[L]$ is a semivector space with identity $\{e\}$ when $A \oplus B=\{a+b \mid a \in A, b \in B\}$ and $\lambda A=\{\lambda a \mid a \in A\}$, where + stands for vector addition in $L\left(A, B \in[L], \lambda \in R_{+}\right)$. Furthermore, $2[L]$ $\subset[L]$ is also a semivector space and is pointwise convex, i.e., $\{A\}$ is convex for each $A \in \mathcal{2}[L]$. In fact $2[L]$ is the largest pointwise convex subset of $[L]:$ If $A \in[L]$ and $\lambda A \oplus \lambda^{\prime} A \subset A$ for each $\lambda=\left(1-\lambda^{\prime}\right) \in[0,1]$, then $A \subset L$ must be convex.

From here on, $L$ will always be a topological vector space.

Now $\mathfrak{K}[L] \subset[L]$ is a semivector subspace and $\mathcal{K}_{2}[L]$ is the largest pointwise convex semivector subspace of $\mathscr{K}[L]$. Also, the origin $0[L]=0 \mathcal{K}[L]=02[L]$ $=0 K_{2}[L]=\{\{e\}\}$ is singleton. $N$. B. The uniform topology on $\mathcal{K}[L]$ coincides with the finite topology [1, p. 153, 1.1 and p. 160,3.3].

2.1. Proposition. (1) $\mathcal{K}[L]$ is a topological semivector space, locally compact iff $L$ is. (2) The map $\uparrow: x \mapsto\{x\}(x \in L)$ iseomorphically embeds $L$ into the topological semivector subspace $\mathfrak{K} 2[L] \subset \mathcal{K}[L]$.

Proof. (ad (1)). $\mathcal{K}[L]$ is Hausdorff as $L$ is (see [1, p. 164, 4.9.8]) and will be locally compact iff $L$ is locally compact (see [1, p. 164, 4.9.12]). This leaves to show only the continuity of the operations $\oplus$ and $\Psi$ of $\mathcal{K}[L]$. The continuity of vector addition $+: L \times L \rightarrow L$ implies the continuity of the map $\hat{+}:[L \times L]$ $\rightarrow[L]$ defined by $\hat{+}(P)=\{a+b \mid(a, b) \in P\}(P \in[L \times L])$ (see [1, p. 169, 5.9.1]). Thus, the restriction of $\hat{f}$ to the space $\mathscr{B}=\{C \times D \mid C, D \in \mathcal{K}[L]\}$ $\subset \mathcal{K}[L \times L]$ of compact boxes is also continuous. Furthermore, the Cartesian product $\pi(C, D)=C \times D$ is continuous on $\mathscr{K}[L] \times \mathcal{K}[L] \rightarrow \mathscr{B}$ (see Theorem 3 of [3]). Now $\oplus$ is simply the composition $\oplus=\hat{+} \circ \pi: \mathscr{K}[L] \times \mathscr{K}[L] \rightarrow \mathscr{K}[L]$, and so is continuous. Similarly, the continuity of scalar multiplication $R_{+} \times L \rightarrow L$ implies that of scalar multiplication $\Psi: R_{+} \times \mathcal{K}[L] \rightarrow \mathcal{K}[L]$.

$(\operatorname{ad}(2))$. From $(1)$ it follows that the space $\mathscr{K} 2[L] \subset \mathcal{K}[L]$ is a topological semivector space. Now the map $f$ is a homeomorphism [1, p. 155, 2] and is easily checked to be a homomorphism.

2.2 Proposition. (1) K2 $[L]$ is cancellative. (2) In fact, for all $A, B, C \in[L]$, whenever (i) $B$ is bounded and $C$ is closed and convex, or (ii) $B$ is compact and $C$ is open and convex, we have $B \subset B \oplus C \Rightarrow e \in C$ (or, equivalently, $B \oplus A$ $\subset B \oplus C \Rightarrow A \subset C)$.

Proof. (ad (1)). Since TrQ $[L]$ is a pointwise convex (Hausdorff) topological 
semivector space with singleton origin, (1) follows from [2, Theorem 2.11]; it also follows directly from (2)(i), proved below.

(ad (2)). Let $B, C \in[L]$ with $C$ convex.

(i) Assume $B$ bounded and $C$ closed, and that $B \subset B \oplus C$. Now, for any $b(i) \in B$, we have $b(i)=b(i+1)+c(i+1)$ for some $b(i+1) \in B$ and $c(i+1) \in C(i=0,1,2, \ldots)$, so that $b(0)-b(n)=\sum_{N} c(i)(N=\{1, \ldots, n\} ; n$ $=1,2, \ldots)$ and, denoting $\bar{c}(n)=n^{-1} \sum_{N} c(i)$, the convexity of $C$ gives $\bar{c}(n) \in C$, while the boundedness of $B$ implies that $\bar{c}(n)=n^{-1}(b(0)-b(n))$ converges to $e$ as $n$ goes to $\infty$. As $C$ is closed, the limit point $e \in C$.

(ii) Assume $B$ compact and $C$ open, and that $B \subset B \oplus C$. Denoting $C_{b}=\{b\} \oplus C$ for every $b \in B,\left\{C_{b} \mid b \in B\right\}$ is then an open cover of $B$, admitting a finite subcover $\left\{C_{b(i)} \mid i \in M\right\}$. Now, for every $i \in M$, there is a $j \in M$, such that $b(i) \in\{b(j)\} \oplus C$ and, hence

$$
\sum_{N} b(i) \in \sum_{N}(\{b(j)\} \oplus C)
$$

for some subset $N \subset M$, where $\Sigma$ is to $\sum$ as $\oplus$ is to + . As $C$ is convex, we may write

$$
\sum_{N}(\{b(j)\} \oplus C)=\left(\sum_{N} b(j)\right) \oplus n C
$$

(see [2, p. 122]). Denoting $\bar{b}=n^{-1} \sum_{N} b(j)$, where $n=\# N$, we therefore have $\bar{b} \in\{\bar{b}\} \oplus C$, whereby $e \in C$.

(To see the claimed equivalence, first note that, if $B \oplus A \subset B \oplus C \Rightarrow A$ $\subset C$ for all $A \in[L]$, then the case of $A=\{e\}$ yields $B \subset B \oplus C \Rightarrow e \in C$. For the rest, assume $B \subset B \oplus C \Rightarrow e \in C$ whenever (i) or (ii), and take any $A \in[L]$ with $B \oplus A \subset B \oplus C$. Pick any $a \in A$, so that $B \oplus\{a\} \subset B \oplus C$, hence $B \subset B \oplus C \oplus\left\{a^{-}\right\}$, where $a^{-}$is the additive inverse of $a$. Now $C$ $\oplus\left\{a^{-}\right\}$is closed and convex (resp., open and convex) whenever $C$ is so. Thus, whether (i) or (ii) is the case, $e \in C \oplus\left\{a^{-}\right\}$, i.e., $a \in C$, showing $A \subset C$.)

2.3 THEOREM. If $X \subset L$ is nonempty compact and convex, then K2 $[X]$ $\subset$ K2 $[L]$ is (nonempty) compact and convex.

Proof. Let $X \subset L$ be nonempty compact and convex. The uniform topology which the (uniform space) $X$ determines for $\mathscr{K}[X]$ yields $\mathscr{K}[X]$ compact Hausdorff, since $X$ is compact Hausdorff (see [1, p. 160, 3.3 and p. $164,4.9 .12])$. Furthermore, $\mathcal{K}[X]$ inherits the same topology as a subspace of $\mathscr{K}[L]$ as it receives from $X$ (see $[1$, p. 167, 5.2.3 and 5.2.3']), so that $\mathscr{K}[X] \subset \mathscr{K}[L]$ is compact Hausdorff.

Now $\mathscr{K} 2[X] \subset \mathcal{K}[X]$ is clearly nonempty and convex, since $X$ is so. This leaves only to show that $K_{2}[X] \subset \mathcal{K}[X]$ is closed. To that end, let $\mathscr{F}$ be a converging filterbase in $K_{2}[X]$. Since $\mathcal{K}[X]$ is compact Hausdorff, the limit point, say $Q$, is unique and $Q \in \mathscr{K}[X]$. We show that $Q$ is also convex.

For each $\lambda \in[0,1]$, denote $\lambda^{\prime}=(1-\lambda)$ and define the map $\Omega_{\lambda}$ on $\mathscr{K}[X]$ through $\Omega_{\lambda}(P)=\lambda P \oplus \lambda^{\prime} P(P \in \mathscr{K}[X])$. Since scalar multiplication in $L$ is continuous, for each $\lambda \in[0,1], \lambda \mathcal{K}[X] \subset \mathcal{K}[L]$ and $\Omega_{\lambda}$ is a continuous mapping of $\mathscr{K}[X]$ into $\mathscr{K}[L]$; in fact, from the convexity of $X$ one easily sees that $\Omega_{\lambda}(\mathcal{K}[X]) \subset \mathcal{K}[X]$ and that the restriction of $\Omega_{\lambda}$ to $\mathcal{K}[X]$ is nothing but the 
identity map of $\mathcal{K}[X]$. Also, given a $P \in \mathcal{K}[X]$, if $\Omega_{\lambda}(P) \subset P$ for each $\lambda \in[0,1]$, then $P \in K_{K 2}[X]$. Take any $\lambda \in[0,1]$. We show that $\Omega_{\lambda}(Q)=Q$. Let $\mathscr{T} \subset \mathcal{K}[X]$ be any nbd of $\Omega_{\lambda}(Q) \in \mathcal{K}[X]$. As $\Omega_{\lambda}$ is continuous, there is a nbd $\mathscr{C} \subset \mathcal{K}[X]$ of $Q \in \mathcal{K}[X]$ such that $\Omega_{\lambda}(\mathcal{Q}) \subset \mathcal{V}$. As $\underline{\mathscr{F}}$ converges to $Q$, there

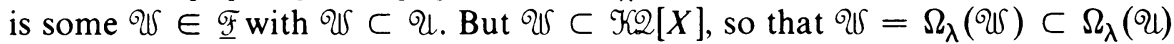
$\subset \mathfrak{V}$. This shows that $\underline{\mathscr{F}}$ converges to $\Omega_{\lambda}(Q)$; and, the limit point being unique, $\Omega_{\lambda}(Q)=Q$. Then $Q \in K_{K}[X]$, showing that $K_{2}[X]$ is closed and completing the proof.

3. Embedding $T_{2}[L]$ in a topological vector space. A subset of the semivector space $[L]$, to be embeddable in a vector space, must clearly be pointwise convex and cancellative. Now the largest pointwise convex set in $[L]$ is $2[L]$, but clearly $2[L]$ fails to be cancellative and is, therefore, not embeddable in a vector space. On the other hand, we have just extended the operations of $L$ to K2 $[L]$ (see 2.1), and this is a topological semivector space which is both pointwise convex and cancellative (2.2). In standard fashion (see also $[2,2.9]$ ) we embed it in

The real vector space $\mathcal{L}$ : Denoting $\delta=K_{2}[L] \times K_{2}[L]$, equip $\delta$ with coordinatewise addition $(A, B) \oplus(C, D)=(A \oplus C, B \oplus D)$ and define the equivalence relation $\mathcal{S} \subset \mathcal{S}$ through $(A, B) \mathcal{S}(C, D) \Leftrightarrow A \oplus D=B \oplus C$, so that $\mathcal{S}$ is a semigroup congruence and the quotient $\mathcal{L}=\delta / \mathcal{G}$ is a group. Denote the equivalence class of $(A, B)$ by $[A, B]$, and define scalar multiplication $\psi: R \times E$ $\rightarrow \mathfrak{L}$ by setting $\psi(\lambda,[A, B])=[\lambda A, \lambda B]$ if $\lambda \geqslant 0$ and $\psi(\lambda,[A, B])=[|\lambda| B$, $|\lambda| A]$ if $\lambda \leqslant 0$. Now $\mathcal{L}$ is a real vector space and the map $g$ which sends each $A \in K_{2}[L]$ to the equivalence class $[2 A, A] \in \mathcal{L}$ is an algebraic isomorphism embedding $K_{2}[L]$ into $\mathcal{L}$. Evidently, $\mathcal{E}$ is, up to an isomorphism, the smallest vector space in which $K_{2}[L]$ may be algebraically embedded. $N$. B. Clearly, $[A, A]=[B, B]$ for all $A, B \in \mathcal{K} \mathcal{K}[L]$, and this equivalence class is the identity element of $\mathcal{L}$.

From here on $L$ will always be locally convex.

We now take a fundamental system $\mathscr{Q}=\left\{U_{\alpha} \mid \alpha \in \mathscr{Q}\right\}$ of symmetric open convex nbds of the identity $e$ in $L$, and for $\mathcal{L}$ we define

The topology J: For each $\alpha \in \mathbb{Q}$, declare $\mho_{\alpha}=\left\{[A, B] \in \mathcal{L} \mid B \subset A \oplus U_{\alpha}, A\right.$ $\left.\subset B \oplus U_{\alpha}\right\}$ to be an open nbd of the identity element $[A, A]$ of $\mathcal{L}$; and, for each $[P, Q] \in \mathcal{L}$, declare $[P, Q] \oplus \mathcal{W}_{\alpha}$ to be an open nbd of $[P, Q]$. (We check that, given $[A, B] \in \mathcal{W}_{\alpha}$ and $(C, D) \in[A, B], \quad D \subset C \oplus U_{\alpha}$ and $C \subset D \oplus U_{\alpha}$ : As $(C, D) \in[A, B]$, we have $A \oplus D=B \oplus C$, while $[A, B] \in \mathcal{W}_{\alpha}$ implies $A$ $\subset B \oplus U_{\alpha}$, whereby $A \oplus D \subset B \oplus D \oplus U_{\alpha}$, so that $B \oplus C \subset B \oplus D \oplus U_{\alpha}$, from which 2.2(2)(ii) implies $C \subset D \oplus U_{\alpha}$; similarly, $D \subset C \oplus U_{\alpha}$.)

3.1. THEOREM. (1) Lequipped with the topology $\sigma$ is a topological vector space, and (2) g embeds K2[L] iseomorphically in $\mathrm{L}$.

Proof. $(\operatorname{ad}(1))$. To see that the family $\mathscr{W}=\left\{\mathscr{W}_{\alpha} \mid \alpha \in \mathcal{Q}\right\}$ is a local base for

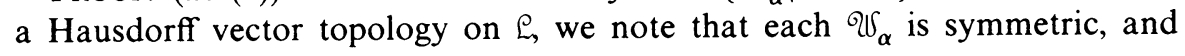
check that:

(i) For each pair $\alpha, \beta \in \mathbb{Q}$, there is a $\gamma \in \mathbb{Q}$ such that $\mathscr{W}_{\gamma} \subset \mathscr{W}_{\alpha} \cap \mathscr{W}_{\beta}$ : Choose $\gamma \in \mathbb{Q}$ such that $U_{\gamma} \subset U_{\alpha} \cap U_{\beta}$. 
(ii) For each $\alpha \in \mathbb{Q}$, there is a $\beta \in \mathbb{Q}$ such that $\mathscr{W}_{\beta} \oplus \mathscr{W}_{\beta} \subset \mathscr{W}_{\alpha}$ : Choose $\beta \in \mathbb{Q}$ such that $U_{\beta} \oplus U_{\beta} \subset U_{\alpha}$.

(iii) For each $\alpha \in Q$, there is a $\beta \in Q$ such that $\lambda \mho_{\beta} \subset \mho_{\alpha}$ for each scalar $\lambda \in R$ with $|\lambda| \leqslant 1$ : Choose $\beta \in \mathbb{Q}$ such that $\lambda U_{\beta} \subset U_{\alpha}$ for each $\lambda \in R$ with $|\lambda| \leqslant 1$.

(iv) Given any $[A, B] \in \mathcal{L}$ and $\alpha \in \mathcal{Q}$, there is a $\lambda \in R$ such that $[A, B]$ $\in \lambda \mho_{\alpha}$ : Taking any $b \in B$, for each $a \in A$ find $\lambda_{a} \in R$ such that $a \in \lambda_{a} U_{\alpha}$ $\oplus\{b\}$, where we may assume $\lambda_{\alpha}>0$ since $U_{\alpha}$ is symmetric. Then, for each $a \in A, a \in \lambda_{a} U_{\alpha} \oplus B$, and so $\left\{\lambda_{a} U_{\alpha} \oplus B \mid a \in A\right\}$ is an open cover of $A$ and, since $A \subset L$ is compact, there is a finite subcover $\left\{\lambda_{a(i)} U_{\alpha} \oplus B \mid i=1, \ldots, m\right\}$. Defining $\lambda_{A}=\operatorname{Max}\left\{\lambda_{a(1)}, \ldots, \lambda_{a(m)}\right\}$, now $A \subset \lambda_{A} U_{\alpha} \oplus B$. Finding $\lambda_{B}$ in similar fashion and setting $\lambda=\operatorname{Max}\left\{\lambda_{A}, \lambda_{B}\right\}$ we see that $[A, B] \in \lambda \mho_{\alpha}$.

(v) $\cap_{\mathbb{Q}} \mathcal{W}_{\alpha}=\{[A, A]\}$ (where $[A, A]$ is the identity element of $\mathcal{L}$ ): $[A, A]$ $\in \cap_{\mathbb{Q}} \mathscr{W}_{\alpha}$, since $[A, A] \in \mathcal{W}_{\alpha}$ for each $\alpha \in \mathbb{Q}$. On the other hand, if $B, C$ $\in K_{2}[L]$ are distinct, then there is a $\beta \in \mathbb{Q}$ such that $B \nsubseteq C \oplus U_{\beta}$ or $C$ $\mathbb{} \subset \oplus U_{\beta}$, so that $[B, C] \notin \mathcal{W}_{\beta}$ and $[B, C] \notin \cap_{\mathbb{Q}} \mathscr{w}_{\alpha}$.

$(\operatorname{ad}(2))$. Having already seen that $\mathrm{g}$ is an algebraic isomorphism, all we need to check here is that $\mathrm{g}$ is continuous and open. A basic open nbd of an element $P \in \mathfrak{K} 2[L]$ is of the form $\mathcal{U}_{\alpha}(P)=\left\{Q \in K_{2}[L] \mid P \subset Q \oplus U_{\alpha}, Q \subset P\right.$ $\left.\oplus U_{\alpha}\right\}(\alpha \in \mathbb{Q})$. A basic open nbd of $g(P)=[2 P, P] \in \mathcal{L}$ according to the subspace topology of $g(\mathcal{K} 2[L])$ determined by $\mathcal{T}$ is of the form $\mathcal{W} \int_{\alpha}^{\prime}(P)$ $=\left([2 P, P] \oplus W_{\alpha}\right) \cap g(\mathcal{K} 2[L])(\alpha \in \mathbb{Q})$. What we actually show now is the formula $g\left(\vartheta_{\alpha}(P)\right)=\vartheta_{\alpha}^{\prime}(P)$.

Let $[2 Q, Q] \in g\left(\mathcal{U}_{\alpha}(P)\right)$, so that $P \subset Q \oplus U_{\alpha}$ and $Q \subset P \oplus U_{\alpha}$. Let

$$
[A, B]=[2 Q, Q] \oplus[P, 2 P]=[2 Q \oplus P, Q \oplus 2 P]
$$

so that $A \oplus Q \oplus 2 P=B \oplus 2 Q \oplus P$, i.e., $A \oplus P=B \oplus Q$. Now $A \oplus P \subset A$ $\oplus Q \oplus U_{\alpha}$, so we have $B \oplus Q \subset A \oplus Q \oplus U_{\alpha}$, and 2.2(2)(ii) then yields $B \subset A \oplus U_{\alpha}$. Similarly, $A \subset B \oplus U_{\alpha}$, so that $[A, B] \in \mathscr{Q} \int_{\alpha}$ and $[2 Q, Q]$ $=[2 P, P] \oplus[A, B] \in \mathcal{Q} \int_{\alpha}^{\prime}(P)$, i.e., $g\left(\mathcal{U}_{\alpha}(P)\right) \subset \mathcal{Q S}_{\alpha}^{\prime}(P)$. Now take any element of $\mathcal{W}_{\alpha}^{\prime}(P)$, i.e., a point

$$
[2 Q, Q]=[2 P, P] \oplus[A, B]=[2 P \oplus A, P \oplus B]
$$

with $A \subset B \oplus U_{\alpha}$ and $B \subset A \oplus U_{\alpha}$. Then $2 Q \oplus P \oplus B=Q \oplus 2 P \oplus A$, so that 2.2(2)(ii) gives $Q \oplus B=P \oplus A \subset P \oplus B \oplus U_{\alpha}$ and $Q \subset P \oplus U_{\alpha}$. Similarly, $P \subset Q \oplus U_{\alpha}$, so $Q \in \mathcal{U}_{\alpha}(P)$ and $[2 Q, Q]=g(Q) \in g\left(\mathcal{U}_{\alpha}(P)\right)$, showing $\mathcal{W}_{\alpha}^{\prime}(P) \subset g\left(\mathcal{U}_{\alpha}(P)\right)$. We conclude that $g\left(\mathcal{U}_{\alpha}(P)\right)=\mathcal{W}_{\alpha}^{\prime}(P)$, and this completes the proof.

\subsection{THEOREM. 2 with the topology $\mathcal{T}$ is locally convex.}

Proof. W.l.o.g., we may assume that, for each $\alpha \in \mathbb{Q}, U_{\alpha}$ is convex, circled, and radial at $e$ and that, for each nonzero $\lambda \in R, \lambda U_{\alpha} \in \mathcal{Q}$. Let $\alpha \in \mathbb{Q}$. It is straightforward to check that (i) $2 \int_{\alpha}$ is circled and (ii) for each nonzero $\lambda \in R, \lambda \mathscr{W}_{\alpha} \in \mathscr{W}$. To check that (iii) $\mathscr{W}_{\alpha}$ is convex, let $[A, B],[C, D]$ $\in \mathcal{W}_{\alpha}$ and $\lambda=\left(1-\lambda^{\prime}\right) \in[0,1]$. Now $\lambda[A, B] \oplus \lambda^{\prime}[C, D]=\left[\lambda A \oplus \lambda^{\prime} C, \lambda B\right.$ $\left.\oplus \lambda^{\prime} D\right]$; and, since $U_{\alpha}$ is convex, we have $\lambda U_{\alpha} \oplus \lambda^{\prime} U_{\alpha}=U_{\alpha}$. Now $[A, B],[C, D]$ $\in \mathcal{W}_{\alpha}$ says $A \subset B \oplus U_{\alpha}$ and $C \subset D \oplus U_{\alpha}$, so that 


$$
\lambda A \oplus \lambda^{\prime} C \subset \lambda B \oplus \lambda^{\prime} D \oplus \lambda U_{\alpha} \oplus \lambda^{\prime} U_{\alpha} .
$$

Similarly, $\lambda B \oplus \lambda^{\prime} D \subset \lambda A \oplus \lambda^{\prime} C \oplus U_{\alpha}$. Thus, $\left[\lambda A \oplus \lambda^{\prime} C, \lambda B \oplus \lambda^{\prime} D\right] \in \mathcal{W}_{\alpha}$, showing that $\mho_{\alpha}$ is convex. This in conjunction with (iv) in the proof of 3.1(1) implies that (iv) $\mathscr{W}_{\alpha}$ is radial at the identity element $[A, A]$ of $\mathcal{E}$. Thus, $\mathcal{W}$ is a local base for a (unique) locally convex topology in $\varrho$.

3.3 THEOREM (RÅDSTRÖM [4]). (1) $巳$ with the topology $\sigma$ is normable iff $L$ is normable, and (2) if $L$ is normed, $\mathcal{L}$ admits a norm for which $f$ and g are isometries.

Proof. (ad (1)). "Only if" is obvious from the conjunction of 2.1(2) and 3.1(2). To see "if", assume that $L$ is normed by a norm $\rho$, so that $V$ $=\{x \in L \mid \rho(x)<1\}=U_{\alpha}$ for some $\alpha \in \mathbb{Q}$. Thus,

$$
\mathcal{Q} \int_{\alpha}=\{[A, B] \in \mathcal{L} \mid A \subset B \oplus V, B \subset A \oplus V\} \in \mathcal{W} \text {. }
$$

Since $V$ is radial at the origin, circled, convex and bounded, one easily checks (see also the proof of 3.1(1)) that $\mathscr{W}_{\alpha}$ has these properties too, so that (the Hausdorff space) $\mathcal{L}$ is normable, proving (1).

$(\operatorname{ad}(2))$. In fact, the Minkowski functional $\rho^{*}$ of $\mho_{\alpha}$ is a norm for $\mathcal{E}$ and, computing that $\rho^{*}[2 P, P]=\operatorname{Sup}_{P} \rho(p)$ for each $P \in \mathcal{K} 2[L]$, one easily sees $f$ and $g$ to be isometries.

ACKNOWLEDGEMENT. The authors thank the International Institute of Management for inviting Prem Prakash to West Berlin, which made it possible for them to reconvene and write this paper.

POST SCRIPTUM (ADDED IN PROOF). The authors are grateful to Professor L. Drewnowski, who not only indicated a gap (due to an erroneous statement and "proof" of a proposition occupying the place of 2.2) in an earlier draft of this paper, but also brought to their attention the work of $\mathbf{R}$. Urbański ( $A$ generalization of the Minkowski-Rådström-Hörmander theorem, Bull. Acad. Polon. Sci. Sér. Sci. Math. Astronom. Phys., forthcoming 1976) embedding the class of all (nonempty) bounded closed and convex subsets of $L$ in a topological vector space even when $L$ fails to be locally convex. It would be of interest to compare the methods of this work with those used here.

\section{REFERENCES}

1. Ernest Michael, Topologies on spaces of subsets, Trans. Amer. Math. Soc. 71 (1951), 152-182. MR 13, 54.

2. Prem Prakash and Murat R. Sertel, Topological semivector spaces: Convexity and fixed point theory, Semigroup Forum 9 (1974), 117-138. MR 51, 1556.

3. - On the continuity of Cartesian product and factorisation, Discussion Paper no. 82, The Center for Mathematical Studies in Economics and Management Science, Northwestern University, Evanston, Ill., 1974. (Also issued as Preprint Series No. I/ 74-16, Easter 1974, International Institute of Management, D-1000 Berlin 33, Griegstrasse 5.)

4. Hans Rådström, An embedding theorem for spaces of convex sets, Proc. Amer. Math. Soc. 3 (1952), 165-169. MR 13, 659.

Graduate School of Management, Northwestern University, Evanston, Illinois 60201 